\title{
Application Analysis of GPS Positioning Technology and GIS Based on Metamaterial in Forestry Engineering
}

\author{
Shuhui Jiang, ${ }^{1,}$, Qin Zong ${ }^{1, b}$ and Xinjie Deng ${ }^{1, c}$ \\ ${ }^{1}$ Chongqing Jianzhu College, Chongqing 400072, China. \\ ajsh0529@126.com, b576036493@qq.com, c1499548393@qq.com
}

\begin{abstract}
Metamaterial refers to artificial composite materials that exhibit extraordinary physical properties that are not found in natural materials. The extraordinary characteristics of metamaterials are derived from the physical response caused by artificially prepared, special non-uniform intercalated structures. Under the rapid development of society and the continuous advancement and development of science and technology, GIS and GPS GIS technology as spatial information analysis technology has begun to develop rapidly and widely. The frequency response characteristics of metamaterials can be adjusted in a wide range according to their structural parameters, so we can design them to work in the frequency range we need through the basic unit structure parameters of metamaterials. Compared with the existing information management methods, the introduction and application of geographic information system technology can provide strong spatial data processing, analysis, query and maintenance capabilities for afforestation project management, and can provide an effective working platform and modern information technology support for obtaining information and decision analysis in the management process of forestry major ecological engineering projects. Based on metamaterials, starting from the advantages of GIS, this paper focuses on analyzing the specific application of GIS in forestry engineering planning and design, and systematically introduces GIS software application technology. To realize the digitalization and networking of forestry projects so as to play a more active role in improving the modern management level of forestry ecological projects in China through the application and demonstration of high and new technologies.
\end{abstract}

\section{Introduction}

With the establishment and implementation of forestry development strategy based on ecological construction, the State Forestry Administration, proceeding from the objective needs of economic and social development for forestry and the national conditions of forestry, follows the natural and economic laws, and centers on the overall objectives and tasks of forestry construction in the new period, has made significant strategic adjustments to the distribution of forestry productivity in China [1]. GPS is the abbreviation of Global Positioning System (GPS). With the development of science and technology and the opening of GPS technology, GPS technology has been applied in national military, surveying and mapping, resource exploration, transportation, water conservancy, meteorology, land, forestry and other industries [2]. Using GIS technology, the survey data, forestry various demarcation lines, professional survey data, etc. are organically combined and applied in various forestry activities such as forest resource survey, resource analysis and forest management. As a technical system, GIS technology is a combination of part data and attribute data. The main purpose is based on the database of measured data, and its position in engineering measurement has become more and more important [3]. The high-tech GIS, which is a combination of computer technology and spatial data, contains various advanced functions for processing geographic information, but its basic functions are data collection, management, processing, analysis and output. The application of GIS geographic information system technology is more, which promotes the intelligent management of forestry resources and data, and makes resource management more uniform and orderly, which provides a more scientific basis for the planning and implementation of engineering projects.

\section{Methodology}

By combining GPS with geographic information system and satellite remote sensing technology, an efficient, real-time and practical forest fire information management system can be established. Once a forest fire occurs, only the fire point coordinates obtained by remote sensing technology can be input [4]. Designed with a handheld GPS receiver combined with the spatial analysis function of GIS, the time and coordinates of 
each inspection by the inspector are recorded in the GPS receiver, and the data is downloaded to the GIS management system for analysis within a prescribed time. The super-material structure composed of short wires can be simulated and analyzed, and the high absorption of vertical incident electromagnetic waves can be completed in the narrow band. The excellent absorbing properties and the easy adjustment of electromagnetic parameters are widely used [5]. Start the GPS, wait for the GPS to search for the island satellite signal, and place the GPS on the ground where it needs to be located, waiting for about 6 minutes. In addition, you have the flexibility to convert maps to a variety of formats. At the same time, modern GIS technology has many functions such as error correction, projection transformation, vectorization of maps and seamless mosaic of maps. This shows that the metamaterial absorber and the free space achieve good impedance matching, and most of the electromagnetic waves enter the absorber [6]. If the imaginary part of the refraction parameter is maximal under this condition. In this structure, the GPS receiver receives satellite signals, saves or transmits them to the management center after calculation, and holds GPS data in the local computer. Due to the differences in site conditions within the patches, the survival rate and preservation rate after afforestation are different, and patches of afforestation land are formed during afforestation design.

In recent years, the improvement of commercial digital cameras and the development of image processing technology make it possible to use commercial digital cameras as image sensors for afforestation monitoring. According to previous related research, remote sensing image data with a resolution of less than $2 \mathrm{~m}$ can be used to monitor forest classes, small classes and even single trees, thereby improving the accuracy and efficiency of afforestation monitoring. Table 1 below shows the statistical results of the tree height and crown sample survey data of the annual saplings of the main afforestation species in the forest area.

Table 1 Statistical results of tree height and crown width sample survey data of annual saplings of main afforestation trees in forest areas

\begin{tabular}{|c|c|c|c|}
\hline Tree species & $\begin{array}{c}\text { Tree height mean } \\
(\mathrm{cm})\end{array}$ & $\begin{array}{c}\text { Minimum crown } \\
\text { size }\left(\mathrm{cm}^{2}\right)\end{array}$ & $\begin{array}{c}\text { Crown mean } \\
\text { value }\left(\mathrm{cm}^{2}\right)\end{array}$ \\
\hline Mountain peach & 28.4 & $40 \times 30$ & $45 \times 47$ \\
\hline Hippophae rhamnoides & 39.6 & $14 \times 8$ & $38 \times 41$ \\
\hline Locust & 37.4 & $11 \times 10$ & $29 \times 32$ \\
\hline
\end{tabular}

The GPS management center is the main construction content of the system. The sub-modules such as inspection line, equipment parameters, vehicle and personnel management can realize the management of the inspection track playback and various equipment detection data. Therefore, the adjustment of the magnetic resonance is quite complicated, and the absorbing property of the metamaterial is weakened by the excitation of the normally incident electromagnetic wave. At the same time, the plot coordinates and survey data stored in the GPS receiver can also directly form a database through data conversion, which is conducive to the summary statistics of the data. Using hand-held GPS navigation function to cut various boundary lines can measure the area of forest land. Secondly, quickly search for the established target, simulate listing on the line at the same time, and configure and retrieve the existing listing function. Because GIS database has a large amount of data, spatial data and attribute data are inseparable, and spatial data have significant topological structure and other characteristics. It enables scientific and rational forestry ecological construction planning, timely and accurately grasp the dynamics of forestry ecological engineering construction and its possible ecological, social and economic benefits, and carry out decision-making, monitoring and evaluation of forestry ecological engineering construction. One of the most important concepts of metamaterials is the ingenious use of key physical scales in materials. Some physical properties presented in materials are often related to the key physical scales in material structures. Determine and eliminate the drift coordinate points. The process of interpretation and processing is as follows: searching for coordinate header marks, interpreting coordinate data, eliminating unreliable data, and transforming, displaying and storing data format.

Based on GIS technology, the batch operation function of ERDAS software is used to automatically complete the image processing of afforestation subcompartments on slopes, and the number of saplings is obtained from the attributes table of each quadrate vector layer. The average value is obtained, and the survival rate of the corresponding small class is obtained by dividing the design number of the small class. The results of comparison with the field sample survey are shown in Table 2 and Figure 1.

Table 2 Comparison of digital close-range image monitoring and field sample survey monitoring results

\begin{tabular}{|c|c|c|}
\hline Image number & $\begin{array}{c}\text { Survival rate of image } \\
\text { monitoring }(\%)\end{array}$ & $\begin{array}{c}\text { Survival rate of quadrat } \\
\text { survey(\%) }\end{array}$ \\
\hline 1 & 78 & 73 \\
\hline 2 & 81 & 79 \\
\hline
\end{tabular}




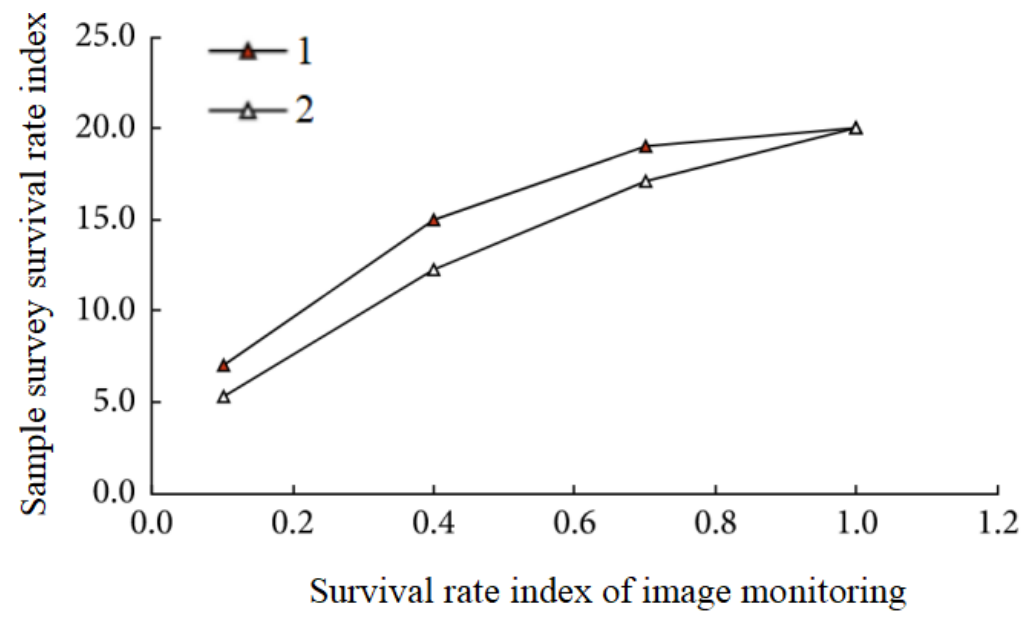

Figure 1 Comparison of digital close-range image monitoring and field sample

With the development of GIS (Geographic Information System), three-dimensional geographic information systems are gradually being used in forestry operations. The three-dimensional geographic information system can truly reproduce the topography of the forest area, providing an ideal space support for us to fully understand the forest resource environment. Modern GIS technology can draw electronic maps and maps, and can flexibly convert maps into multiple formats. System management can also be performed on large-capacity libraries. Auxiliary mapping helps to improve the design of engineering surveyors. Due to the variety of data types involved in GIS, the quality of the same type may vary widely. Data processing is one of the basic functions of GIS in order to ensure the standardization and unification of system data and establish data files that meet the needs of users. It realizes the regulation and control of the electromagnetic wave movement state through the dielectric periodic structure of the material on the wavelength scale. In view of the wonderful characteristics of metamaterials, metamaterials are used to achieve high absorption of electromagnetic waves, which opens up a new field for the study of absorbing materials. The system can precisely coordinate transformation and registration of vector layers. The coordinates of national standard and non-standard vector graphics can be reduced to geodetic coordinates by coordinate mapping transformation. The role of the field should be utilized to construct information network throughout the forestry system, change the way of traditional forestry work and management, and realize the transformation from "traditional forestry going up mountain" to "modern forestry going online". In addition, GPS can also simply and accurately measure the area of affected forest land, estimate the loss of forest fire, and output forest fire disaster maps, fire risk grade maps and so on, which is of great significance to forest fire prevention.

\section{Result Analysis and Discussion}

Data processing is the core of GIS technology application, and how the effectiveness of forestry planning is largely determined by data processing. From this point of view, it is very important to do a good job of data processing. Both the dielectric constant and the magnetic permeability take a negative value at the same time. This material certainly does not exist in nature. But it can be achieved through the design of metamaterials. The combination of multimedia technology and visualization technology has effectively improved the way of transmitting geographic information. Such visualization technology is usually combined with multimedia technology to make the transmission of geographic information different from the previous expressions of graphics, text and tables, and become a multimedia space. Presentation content. It is to automatically establish buffer polygons of various types of elements according to the point, line and surface entities of the database, so as to determine the spatial proximity or proximity of different geographical elements. Among them, information technology refers to the technology used in the production, exchange, transmission, processing and utilization of forestry information based on computer. Therefore, the relevant forestry departments at the national level should attach importance to the application of forestry intelligent technology, increase investment and policy support for forestry work, and ensure the smooth and orderly development of forestry work. It can customize the scale according to the user's requirements, modify and edit the map, and generate various thematic maps and forest maps according to the attributes of subcompartments.

Graphic data acquisition is one of the basic functions of GIS software, with high efficiency and high quality data acquisition function. Therefore, remote sensing image processing technology can be used to classify, recode and convert single image into vector format, and extract surface information such as tree pit and sapling number, etc. by taking close-range images taken by common digital cameras as traditional remote sensing images. In order to perform overlay display analysis with other raster image data or vector graphics with geographic coordinates, it is necessary to carry out geographic registration on the raster images with the above coordinates with known real geographic coordinates. The metamaterial absorber realizes perfect 
impedance matching with free space in a wider frequency range, and the reflectivity of electromagnetic wave decreases slightly with the decrease of short wire width. Because the unsupervised classification is clustered according to the spectral characteristics of the image without human intervention, it has a certain blindness. Therefore, the classification result obtained needs to be classified and processed in combination with the original image. When a new map is created and a data layer is loaded into one of the data, the coordinate system of the first loaded data layer acts as the default coordinate system for the data set. For geometry, the system automatically calculates its area and perimeter by establishing topological relationships, calculates the length of the line features, and determines the position of the point features. Therefore, relevant national forestry departments should pay attention to the application of forestry intelligent technology, increase investment and policy support for forestry work, and ensure the smooth and orderly development of forestry work.

Using a digital camera to capture close-up images to monitor the survival rate of afforestation, on the one hand, it can identify single trees in afforestation and afforestation, determine the survival rate, preservation rate and afforestation quality of afforestation, and carry out afforestation monitoring work more effectively. Of course, this conversion does not affect the data file itself corresponding to the data layer. If a data layer does not have enough coordinate information to achieve coordinate transformation, it is impossible to correctly express it in the map. The application of GIS technology to forestry engineering planning and design can integrate various data factors, which will also enable forestry planning decisions to become more realistic and more reasonable. Through the implementation of GPS application technology, it can improve the work efficiency by about $50 \%$, reduce the labor intensity of field investigators, and eliminate the need to cut down a large number of coordinate trees. If markers for reference are set up in the shooting sub-compartment, geometric correction and projection conversion are carried out on the digital close-range photos by means of digital photogrammetry principle and GIS software, and the obtained image data can be used for geographic information analysis. GIS, as a tool for forest survey and data management, organically combines survey data, basic geographic elements, subcompartment division boundaries and professional survey data to establish geographic information base. Through the use of GIS technology, the spatial data and distribution of forest resources will be transmitted to the staff in time, so that the staff will also be able to understand the forest changes in time, thus improving the management efficiency to a certain extent.

\section{Summary}

The application of GPS and GIS software can accurately and quickly collect and manage geospatial data, complete the rapid entry of graphic data, compile beautiful image results, superimpose and integrate various layers, and output statistical reports. The use of GIS technology for afforestation small-scale auxiliary planning analysis and monitoring has the advantages of quickness, precision and scientificity, which can overcome the inconveniences of traditional afforestation planning work, extensive work of grassroots planning, and human factors. The application of GIS technology in forestry engineering planning has become an inevitable choice for future development. In the future, the use of GIS technology will effectively improve work efficiency, realize scientific management and reduce work costs. With the widespread application of this system, the application level of GIS will be continuously improved, and this system as a primary system can play a very good starting role. In short, under the trend of rapid economic development in our country, the crisis of forest resources is aggravating. Therefore, accelerating the application of GIS system in forestry investigation project is irresistible. Attention must be paid to accelerate innovation and promote forestry development.

\section{Acknowledgement}

Chongqing higher education teaching reform research project, project NO. 163270;

Education and teaching research project of Chongqing Jianzhu College, project NO. 16J1

\section{References}

1. Janowski A, Nowak A, Przyborski $M$, et al. Mobile Indicators in GIS and GPS Positioning Accuracy in Cities[J]. Lecture Notes in Computer Science, 2014, 8537(8537):309-318.

2. Zhang H, Zheng J, Dorr G, et al. Testing of GPS Accuracy for Precision Forestry Applications[J]. Arabian Journal for Science and Engineering, 2014, 39(1):237-245.

3. $\mathrm{Xu} \mathrm{W} \mathrm{H,} \mathrm{Feng} \mathrm{Z} \mathrm{K,} \mathrm{Su} \mathrm{Z} \mathrm{F,} \mathrm{et} \mathrm{al.} \mathrm{An} \mathrm{Automatic}$ Extraction Algorithm for Individual Tree Crown Projection Area and Volume Based on 3D Point Cloud Data[J]. Spectroscopy and Spectral Analysis, 2014, 34(2):465.

4. Amanda S, Kevin M D, Ger D. Analysing Performance Characteristics of Biomass Haulage in Ireland for Bioenergy Markets with GPS, GIS and Fuel Diagnostic Tools[J]. Energies, 2015, 8(10):12004-12019.

5. Wang X, Kostyniuk L, Barnes M. Built Environment and Driving Outcomes: The Case for an Integrated GIS/GPS Approach[J]. International Journal of Applied Geospatial Research, 2014, 5(2):11-29.

6. Cai M, Zou J, Xie J, et al. Road traffic noise mapping in Guangzhou using GIS and GPS[J]. Applied Acoustics, 2015, 87:94-102. 